\title{
Inherent Numerical Instability in Computing Invariant Measures of Markov Chains
}

\author{
Hendrik Baumann, Thomas Hanschke \\ Clausthal University of Technology, Clausthal-Zellerfeld, Germany \\ Email: hendrik.baumann@tu-clausthal.de, thomas.hanschke@tu-clausthal.de
}

How to cite this paper: Baumann, $\mathrm{H}$. and Hanschke, T. (2017) Inherent Numerical Instability in Computing Invariant Measures of Markov Chains. Applied Mathematics, 8, 1367-1385.

https://doi.org/10.4236/am.2017.89101

Received: August 14, 2017

Accepted: September 27, 2017

Published: September 30, 2017

Copyright $\odot 2017$ by authors and Scientific Research Publishing Inc. This work is licensed under the Creative Commons Attribution International License (CC BY 4.0).

http://creativecommons.org/licenses/by/4.0/ (c) (i) Open Access

\begin{abstract}
Invariant measures of Markov chains in discrete or continuous time with a countable set of states are characterized by its steady state recurrence relations. Exemplarily, we consider transition matrices and Q-matrices with upper bandwidth $\mathrm{n}$ and lower bandwidth 1 where the invariant measures satisfy an $(n+1)$-order linear difference equation. Markov chains of this type arise from applications to queueing problems and population dynamics. It is the purpose of this paper to point out that the forward use of this difference equation is subject to some hitherto unobserved aspects. By means of the concept of generalized continued fractions (GCFs), we prove that each invariant measure is a dominated solution of the difference equation such that forward computation becomes numerically unstable. Furthermore, the GCF-based approach provides a decoupled recursion in which the phenomenon of numerical instability does not appear. The procedure results in an iteration scheme for successively computing approximants of the desired invariant measure depending on some truncation level $\mathrm{N}$. Increasing $\mathrm{N}$ leads to the desired solution. A comparison study of forward computation and the GCF-based approach is given for Q-matrices with upper bandwidth 1 and 2 .
\end{abstract}

\section{Keywords}

Invariant Measures of Markov Chains, Inherent Numerical Instability of Linear Difference Equations, Generalized Continued Fractions, Convergence Criteria for Generalized Continued Fractions, Truncation Procedures for Infinite Matrices

\section{Introduction}

This paper is dedicated to discrete-time Markov chains $X=\left(X_{k}\right)_{k>0}$ with a countably infinite set of states (labelled as $0,1,2, \cdots)$ and a stationary one-step 
transition matrix $P=\left(P_{i k}\right)_{0 \leq i, k<\infty}$ of the form

$$
P=\left[\begin{array}{ccccccccc}
P_{00} & P_{01} & P_{02} & \ldots & P_{0 n} & O & O & \ldots & \ldots \\
P_{10} & P_{11} & P_{12} & \ldots & P_{1 n} & P_{1, n+1} & O & \ldots & \ldots \\
O & P_{21} & P_{22} & \ldots & P_{2 n} & P_{2, n+1} & P_{2, n+2} & O & \ldots \\
O & O & P_{32} & \ldots & P_{3 n} & P_{3, n+1} & P_{3, n+2} & P_{3, n+3} & \\
\ddots & \ddots & \ddots & & \ddots & \ddots & \ddots & \ddots & \ddots
\end{array}\right]
$$

with $n \geq 1$ and $P_{k, k+n} \neq 0$ for at least one $k \in \mathbb{N}_{0}=\{0,1,2, \cdots\}$. In addition we assume that $X$ is irreducible and recurrent. Notice that the irreducibility of $X$ implies

$$
P_{k, k-1} \neq 0 \text { for } k=1,2, \cdots
$$

For a recurrent irreducible Markov chain, the system

$$
\left\{\begin{array}{l}
x_{k}=\sum_{i=0}^{\infty} x_{i} P_{i k} \quad(k=0,1,2, \cdots) \\
x_{0}=1, x_{k} \geq 0 \quad(k=1,2, \cdots)
\end{array}\right.
$$

has a unique solution which is strictly positive, see Karlin and Taylor ([1], chapter 11). Every constant positive multiple of $x=\left(x_{0}, x_{1}, \cdots\right)$ is called an invariant measure of $X$.

Substituting (1) into (3) and rearranging yields the $(n+1)$ th-order homogeneous linear recurrence relation

$$
P_{k+n+1, k+n} X_{k+n+1}+\left(P_{k+n, k+n}-1\right) x_{k+n}+\cdots+P_{k, k+n} X_{k}=0 \quad(k=0,1,2, \cdots)
$$

coupled to the side conditions

$$
\left\{\begin{array}{l}
P_{00} x_{0}+P_{10} x_{1}=x_{0} \\
P_{01} x_{0}+P_{11} x_{1}+P_{21} x_{2}=x_{1} \\
\vdots \\
P_{0, n-1} x_{0}+P_{1, n-1} x_{1}+\cdots+P_{n, n-1} x_{n}=x_{n-1}
\end{array}\right.
$$

From the theory of linear difference equations (see Miller [2]) it is known that there exist $n+1$ linearly independent functions $x^{(0)}, x^{(1)}, \cdots, x^{(n)}$ defined on $\mathbb{N}_{0}$ which take on prescribed values at $k=0,1, \cdots, n$ and satisfy the homogeneous recurrence relation (4) for all $k \geq 0$.

Since $P_{k+n+1, k+n} \neq 0$ for all $k$ the desired solution $\left(x_{k}\right)_{k=0}^{\infty}$ is uniquely determined by its initial values $x_{0}, x_{1}, \cdots, x_{n}$ and (5). An application of the recurrence relation (4) then should directly lead to the desired solution $x$. But unfortunately forward computation of $x$ by means of the homogeneous equation (4) is not a meaningful procedure. In the sequel it is shown that the solution space $S$ of the recurrence relation (4) is the direct sum

$$
S=S_{1} \oplus S_{2}
$$

of two subspaces $S_{1}$ and $S_{2}$ with the property, that every solution $y \in S_{2}$ dominates over every solution $x \in S_{1}$, i.e.

$$
\lim _{k \rightarrow \infty} \frac{x_{k}}{y_{k}}=0 .
$$


In addition, we have $\operatorname{dim} S_{1}=n, \operatorname{dim} S_{2}=1$ and $x \in S_{1}$.

As pointed out by Cash [3], Gautschi [4] [5], Lozier [6] and Mattheij [7], forward computation of a dominated solution becomes numerically unstable. To avoid numerical instability, it is therefore necessary to construct a decoupled recursion in which the dominant solution $y \in S_{2}$ does not appear. In the sequel, it is shown that with each transition matrix $P$ of the form (1) one can associate a set of so-called generalized continued fractions (GCFs) being convergent if the underlying Markov chain is irreducible and recurrent. It turns out that the limits of these fractions coincide with the coefficients of the desired decoupled recursion.

The phenomenon of inherent instability has been first recognized in connection with the computation of higher transcendental functions such as Bessel and associated Legendre functions (see Gautschi [4] and Wimp [8]). Our experience seems to indicate that also most of the recurrence relations arising from stochastic modelling are subject to numerical instability and require special attention. Transition matrices and $Q$-matrices with lower bandwidth $n$ and upper bandwidth 1 have been discussed in [9].

Remark. Most of our considerations concerning the computation of invariant measures of Markov chains may be extended to more general infinite systems of equations. In Section 3, we will point out the properties of invariant measures which we explicitly used.

\section{Generalized Continued Fractions and Linear Difference Equations}

This chapter deals with the computation of dominated solutions of homogeneous linear difference equations by means of generalized continued fractions (GCFs). The theory of GCFs was initiated by Jacobi [10] and Perron [11] [12] and played a leading role in different areas of mathematics, especially in number theory, ergodic theory, linear difference equations and Padé approximations.

Defintion 1. A generalized continued fraction (GCF) of dimension $n$ is an $(n+1)$-tuple $\left(a^{(1)}, \cdots, a^{(n)}, b\right)$ of real-valued sequences and a convergence structure as follows. Let $A^{(1)}, \cdots, A^{(n)}, B$ be the principal solutions of the corresponding homogeneous linear difference equation

$$
x_{k+n+1}=b_{k} x_{k+n}+a_{k}^{(n)} x_{k+n-1}+\cdots+a_{k}^{(1)} x_{k}(k=0,1,2, \cdots)
$$

satisfying

$$
\left\{\begin{array}{l}
A_{j}^{(i)}=\delta_{i, j+1}=\left\{\begin{array}{l}
1 \text { for } i=j+1 \\
0 \text { for } i \neq j+1
\end{array}(i=1, \cdots, n ; j=0, \cdots, n)\right. \\
B_{j}=0 \text { for } j=0, \cdots, n-1 \text { and } B_{n}=1 .
\end{array}\right.
$$

The GCF is said to converge iff all the limits

$$
\xi^{(i)}=\lim _{k \rightarrow \infty} A_{k}^{(i)} / B_{k}(i=1, \cdots, n)
$$


do exist.

Convergence of a GCF is indicated by the notation

$$
\left[\begin{array}{c}
\xi^{(1)} \\
\vdots \\
\xi^{(n)}
\end{array}\right] \stackrel{k}{=}\left[\begin{array}{cccc}
a_{0}^{(1)} & a_{1}^{(1)} & a_{2}^{(1)} & \ldots \\
\vdots & \vdots & \vdots & \\
a_{0}^{(n)} & a_{1}^{(n)} & a_{2}^{(n)} & \ldots \\
b_{0} & b_{1} & b_{2} & \ldots
\end{array}\right] .
$$

Terminating a GCF after the $N$-th column we get the so-called $N$-th approximants

$$
\left[\begin{array}{c}
\frac{A_{N+n+1}^{(1)}}{B_{N+n+1}} \\
\vdots \\
\frac{A_{N+n+1}^{(n)}}{B_{N+n+1}}
\end{array}\right]=\left[\begin{array}{cccc}
a_{0}^{(1)} & a_{1}^{(1)} & \cdots & a_{N}^{(1)} \\
\vdots & \vdots & \ddots & \vdots \\
a_{0}^{(n)} & a_{1}^{(n)} & \cdots & a_{N}^{(n)} \\
b_{0} & b_{1} & \cdots & b_{N}
\end{array}\right]
$$

The term "GCF" becomes obvious if forward computation of the $N$-th approximant of a GCF is replaced by the equivalent backward algorithm. It is known that the recurrence relation (6) can be converted into a first order vector recursion of the form

$$
u_{k+1}=W_{k} u_{k} \quad(k=0,1,2, \cdots)
$$

by putting

$$
u_{k}=\left[\begin{array}{c}
x_{k} \\
x_{k+1} \\
\vdots \\
x_{k+n-1} \\
x_{k+n}
\end{array}\right] \text { and } W_{k}=\left[\begin{array}{cccccc}
0 & 1 & 0 & \cdots & 0 & 0 \\
0 & 0 & 1 & \cdots & 0 & 0 \\
\vdots & \vdots & \vdots & \ddots & \vdots & \vdots \\
0 & 0 & \cdots & \cdots & 0 & 1 \\
a_{k}^{(1)} & a_{k}^{(2)} & \cdots & \cdots & a_{k}^{(n)} & b_{k}
\end{array}\right](k=0,1,2, \cdots) .
$$

Comparing (6) and (9) it is seen, that the numerators $A_{N+n+1}^{(1)}, \cdots, A_{N+n+1}^{(n)}$ and the denominators $B_{N+n+1}$ of the GCF appear in the last row of the matrix

$$
W_{N} W_{N-1} \cdots W_{0} \text {. }
$$

Consider now the backward recurrence scheme

$$
{ }_{(N)} v_{k}={ }_{(N)} v_{k+1} W_{k} \quad(k=N, N-1, \cdots, 0)
$$

with initial vector ${ }_{(N)} v_{N+1}=(0, \cdots, 0,1)$. Then

$$
{ }_{(N)} V_{0}={ }_{(N)} V_{N+1} W_{N} W_{N-1} \cdots W_{0}=\left(A_{N+n+1}^{(1)}, \cdots, A_{N+n+1}^{(n)}, B_{N+n+1}\right) .
$$

Writing (10) in expanded form and inserting the structure of $W_{k}$, we get

$$
\begin{gathered}
{ }_{(N)} v_{k}^{(1)}={ }_{(N)} v_{k+1}^{(n+1)} a_{k}^{(1)} \quad(k=N, N-1, \cdots, 0), \\
{ }_{(N)} v_{k}^{(i)}={ }_{(N)} v_{k+1}^{(i-1)}+{ }_{(N)} v_{k+1}^{(n+1)} a_{k}^{(i)} \quad(i=2, \cdots, n, k=N, N-1, \cdots, 0), \\
{ }_{(N)} v_{k}^{(n+1)}={ }_{(N)} v_{k+1}^{(n)}+{ }_{(N)} v_{k+1}^{(n+1)} b_{k} \quad(k=N, N-1, \cdots, 0)
\end{gathered}
$$

or, equivalently 


$$
\left\{\begin{array}{l}
{ }_{(N)} r_{k}^{(1)}:=\frac{{ }_{(N)} v_{k}^{(1)}}{{ }_{(N)}^{(n+1)}}=\frac{a_{k}^{(1)}}{b_{k}+{ }_{(N)} r_{k+1}^{(n)}} \quad(k=N, N-1, \cdots, 0) \\
{ }_{(N)} r_{k}^{(i)}:=\frac{(N) v_{k}^{(i)}}{{ }_{(N)} v_{k}^{(n+1)}}=\frac{a_{k}^{(i)}{ }_{(N)} r_{k+1}^{(i-1)}}{b_{k}+{ }_{(N)} r_{k+1}^{(n)}} \quad(i=2, \cdots, n ; k=N, N-1, \cdots, 0)
\end{array}\right.
$$

with initial values ${ }_{(N)} r_{N+1}^{(i)}=0$ for $i=1, \cdots, n$. Hence

$$
\frac{A_{N+n+1}^{(i)}}{B_{N+n+1}}={ }_{(N)} r_{0}^{(i)} \quad(i=1, \cdots, n)
$$

and

$$
\xi^{(i)}=\lim _{N \rightarrow \infty}(N) r_{0}^{(i)} \quad(i=1, \cdots, n) .
$$

Alternative procedures for computing GCFs are described in [13].

The relations between GCFs and linear difference equations have been first recognized by Perron [14]. Perron's results were generalized by Van der Cruyssen [13], Hanschke [15] [16] and Levrie and Bultheel [17]. The following theorem is due to Van der Cruyssen [13].

Theorem 1. A GCF $\left(a^{(1)}, \cdots, a^{(n)}, b\right)$ converges iff there are $n+1$ linearly independent solutions $x^{(1)}, \cdots, x^{(n)}, y$ of the recurrence relation (6) satisfying

$$
\lim _{k \rightarrow \infty} \frac{x_{k}^{(i)}}{y_{k}}=0 \quad(i=1, \cdots, n)
$$

and

$$
\left|\begin{array}{ccc}
x_{0}^{(1)} & \cdots & x_{n-1}^{(1)} \\
\vdots & & \vdots \\
x_{0}^{(n)} & \cdots & x_{n-1}^{(n)}
\end{array}\right| \neq 0 .
$$

As pointed out by Gautschi [4] [5] and Van der Cruyssen [13], forward computation of a solution $x \in \operatorname{span}\left(x^{(1)}, \cdots, x^{(n)}\right)$ is numerically unstable. Van der Cruyssen [13] establishes that if the limits

$$
\left[\begin{array}{c}
\xi_{l}^{(1)} \\
\vdots \\
\xi_{l}^{(n)}
\end{array}\right]=\left[\begin{array}{cccc}
a_{l}^{(1)} & a_{l+1}^{(1)} & a_{l+2}^{(1)} & \ldots \\
\vdots & \vdots & \vdots & \\
a_{l}^{(n)} & a_{l+1}^{(n)} & a_{l+2}^{(n)} & \ldots \\
b_{l} & b_{l+1} & b_{l+2} & \ldots
\end{array}\right]
$$

exist for all $l \geq 0$, then $x \in \operatorname{span}\left(x^{(1)}, \cdots, x^{(n)}\right)$ iff

$$
x_{l+n}=-\xi_{l}^{(n)} x_{l+n-1}-\cdots-\xi_{l}^{(1)} x_{l} \quad(l=0,1,2, \cdots) .
$$

Combining (11), (12) and (16), one obtains an efficient algorithm (which we will refer to as Van der Cruyssen's algorithm) for approximating the first $L+1$ components $x_{0}, \cdots, x_{L}$ of an element $x \in \operatorname{span}\left(x^{(1)}, \cdots, x^{(n)}\right)$ with prescribed values $x_{0}, \cdots, x_{n-1}$ :

Step 1: Select $N>L-n$ and define ${ }_{(N)} r_{l}^{(i)}$ for $i=1, \cdots, n ; l=0,1, \cdots, N+1$ by 


$$
\begin{gathered}
{ }_{(N)} r_{N+1}^{(1)}=\cdots={ }_{(N)} r_{N+1}^{(n)}=0, \\
{ }_{(N)} r_{l}^{(1)}=\frac{a_{l}^{(1)}}{b_{l}+{ }_{(N)} r_{l+1}^{(n)}}(l=N, N-1, \cdots, 0), \\
{ }_{(N)} r_{l}^{(i)}=\frac{a_{l}^{(i)}+{ }_{(N)} r_{l+1}^{(i-1)}}{b_{l}+{ }_{(N)} r_{l+1}^{(n)}}(i=2, \cdots, n ; l=N, N-1, \cdots, 0) .
\end{gathered}
$$

Step 2: Set ${ }_{(N)} x_{0}=x_{0}, \cdots,{ }_{(N)} x_{n-1}=x_{n-1}$ and

$$
{ }_{(N)} X_{l+n}=-{ }_{(N)} r_{l}^{(n)}{ }_{(N)} X_{l+n-1}-\cdots-{ }_{(N)} r_{l}^{(1)}{ }_{(N)} X_{l}
$$

for $l=0,1, \cdots, L-n$.

Step 3: Increase $N$ until the accuracy of the iterates is within prescribed limits.

For any $l$, the vector $\left({ }_{(N)} r_{l}^{(1)}, \cdots,{ }_{(N)} r_{l}^{(n)}\right)^{\mathrm{T}}$ is an approximant of a GCF. Hence, convergence of the algorithm is related to convergence of GCFs. For the latter, we cite two helpful results.

Theorem 2. (Levrie [18], Perron [12]). The GCF

$$
\left[\begin{array}{cccc}
a_{0}^{(1)} & a_{1}^{(1)} & a_{2}^{(1)} & \ldots \\
\vdots & \vdots & \vdots & \\
a_{0}^{(n)} & a_{1}^{(n)} & a_{2}^{(n)} & \ldots \\
b_{0} & b_{1} & b_{2} & \ldots
\end{array}\right]
$$

converges if it satisfies the so-called dominance condition, i.e.

$$
\left|a_{k}^{(1)}\right|+\left|a_{k}^{(2)}\right|+\cdots+\left|a_{k}^{(n)}\right|+1 \leq\left|b_{k}\right| \text { for all } k \geq 0 .
$$

Theorem 3. (De Bruin [19], Perron [12]). Consider a GCF of the form (8) with vth approximant numerators $A_{v+n+1}^{(i)}(i=1, \cdots, n)$ and denominators $B_{v+n+1}$ and the GCF given by

$$
\left[\begin{array}{ccc}
a_{0}^{(1)} \rho_{0} \rho_{-1} \cdots \rho_{-n} & a_{1}^{(1)} \rho_{1} \rho_{0} \cdots \rho_{1-n} \cdots & a_{v}^{(1)} \rho_{v} \rho_{v-1} \cdots \rho_{v-n} \cdots \\
a_{0}^{(2)} \rho_{0} \rho_{-1} \cdots \rho_{1-n} & a_{1}^{(2)} \rho_{1} \rho_{0} \cdots \rho_{2-n} \cdots & a_{v}^{(2)} \rho_{v} \rho_{v-1} \cdots \rho_{v-n+1} \\
\vdots & \vdots & \vdots \\
a_{0}^{(n)} \rho_{0} \rho_{-1} & a_{1}^{(n)} \rho_{1} \rho_{0} & a_{v}^{(n)} \rho_{v} \rho_{v-1} \\
b_{0} \rho_{0} & b_{1} \rho_{1} & b_{v} \rho_{v}
\end{array}\right]
$$

with $v$ th numerators $\tilde{A}_{v+n+1}^{(i)}(i=1, \cdots, n)$ and denominators $\tilde{B}_{v+n+1}$ where the real numbers $\rho_{-n}, \rho_{-n+1}, \cdots, \rho_{0}, \rho_{1}, \cdots$ are all different from zero. Then

$$
\left.\begin{array}{l}
\tilde{A}_{v+n+1}^{(i)}=\rho_{v} \rho_{v-1} \cdots \rho_{1} \rho_{0} \cdots \rho_{-n+i-1} A_{\nu+n+1}^{(i)} \quad(i=1, \cdots, n) \\
\tilde{B}_{v+n+1}=\rho_{v} \rho_{v-1} \cdots \rho_{0} B_{v+n+1}
\end{array}\right\} v \in \mathbb{N}_{0}
$$

In other words, if one of these two GCFs converges so does the other.

Notice that the GCF (17) may be interpreted as an equivalence transformation of the GCF (12).

\section{Main Results}

To make (4) congruent with (6) we put 


$$
\begin{gathered}
a_{k}^{(i)}=-\frac{P_{k+i-1, k+n}}{P_{k+n+1, k+n}} \quad(i=1, \cdots, n, k=0,1,2, \cdots), \\
b_{k}=-\frac{P_{k+n, k+n}-1}{P_{k+n+1, k+n}} \quad(k=0,1,2, \cdots) .
\end{gathered}
$$

Theorem 4. Let $a_{k}^{(i)}, b_{k}$ be defined as in (18) and (19). The limits

$$
\left[\begin{array}{c}
\xi_{l}^{(1)} \\
\vdots \\
\xi_{l}^{(n)}
\end{array}\right]=\left[\begin{array}{cccc}
a_{l}^{(1)} & a_{l+1}^{(1)} & a_{l+2}^{(1)} & \ldots \\
\vdots & \vdots & \vdots & \\
a_{l}^{(n)} & a_{l+1}^{(n)} & a_{l+2}^{(n)} & \ldots \\
b_{l} & b_{l+1} & b_{l+2} & \ldots
\end{array}\right]
$$

exist for all $l \geq 0$ if the corresponding Markov chain is irreducible and recurrent. In case of convergence the invariant measure is a dominated solution of (6) and satisfies the decoupled recursion

$$
x_{l+n}=-\xi_{l}^{(n)} x_{l+n-1}-\cdots-\xi_{l}^{(1)} x_{l} \quad(l=0,1,2, \cdots) .
$$

Proof. Denote by ${ }_{(N)} P=\left(P_{i k}\right)_{0 \leq i, k \leq N}$ the $(N+1) \times(N+1)$ northwest corner truncation of $P$. Since $P$ is assumed to be irreducible and recurrent we conclude from Seneta [20] [21] [22] that the finite system

$$
{ }_{(N)} h\left({ }_{(N)} I-{ }_{(N)} P\right)=e_{0}
$$

where ${ }_{(N)} I$ is the unit matrix and ${ }_{(N)} e_{0}$ is the vector with unity in the first position and zeros elsewhere has a unique solution ${ }_{(N)} h=\left({ }_{(N)} h_{0},{ }_{(N)} h_{1}, \cdots,{ }_{(N)} h_{N}\right)$ satisfying

$$
\lim _{N \rightarrow \infty} \frac{(N) h_{k}}{(N)}=\frac{x_{k}}{x_{0}} \quad(k=0,1,2, \cdots) .
$$

The vector ${ }_{(N)} h$ satisfies the homogeneous linear difference Equation (6) for $k=0,1, \cdots, N-n$ and is subject to the boundary conditions

$$
\left.\begin{array}{c}
\left(1-P_{00}\right)_{(N)} h_{0}-P_{10(N)} h_{1}=1 \\
-P_{01(N)} h_{0}+\left(1-P_{11}\right)_{(N)} h_{1}-P_{21(N)} h_{2}=0 \\
\vdots \\
-P_{0, n-1(N)} h_{0}-P_{1, n-1(N)} h_{1}-\cdots-P_{n, n-1(N)} h_{n}=0
\end{array}\right\}
$$

and

$$
{ }_{(N)} h_{N+1}=0 .
$$

Notice that the numerators $A_{1}^{(1)}, \cdots, A_{l}^{(n)}$ and the denumerators $B_{l}$ of the GCF (20) satisfy

$$
x_{k+n+1}=b_{k+l} x_{k+n}+a_{k+l}^{(n)} x_{k+n-1}+\cdots+a_{k+l}^{(1)} x_{k} \quad(k=0,1,2, \cdots)
$$

and build up a fundamental system of solutions to Equation (6) for $k \geq l$. 
Hence any solution of the recurrence relation (6) can be expressed in terms of these functions. In view of their initial values

$$
\left\{\begin{array}{l}
A_{l, j}^{(i)}=\delta_{i, j+1}=\left\{\begin{array}{l}
1 \text { for } i=j+1 \\
0 \text { for } i \neq j+1
\end{array} \quad(i=1, \cdots, n ; j=0, \cdots, n)\right. \\
B_{l, j}=0 \text { for } j=0, \cdots, n-1 \text { and } B_{l, l+n}=1 ;
\end{array}\right.
$$

we get

$$
{ }_{(N)} h_{k}={ }_{(N)} h_{l} A_{l, k-l}^{(1)}+\cdots+{ }_{(N)} h_{l+n-1} A_{l, k-l}^{(n)}+{ }_{(N)} h_{l+n} B_{l, k-l} \quad(k=l, l+1, \cdots) .
$$

Choosing $k=N+1$ and utilizing (24) equation (27) reduces to

$$
(N) h_{l+n} B_{l, N-l+1}=-_{(N)} h_{l} A_{l, N-l+1}^{(1)}-\cdots-{ }_{(N)} h_{l+n-1} A_{l, N-l+1}^{(n)} .
$$

Dividing both sides of (28) by $B_{l, N-l+1}$ and ${ }_{(N)} h_{0}$ we formally arrive at

$$
\frac{(N) h_{l+n}}{(N) h_{0}}=-\frac{(N)}{{ }_{(N)} h_{l}} \frac{A_{l, N-l+1}^{(1)}}{B_{l, N-l+1}}-\cdots-\frac{(N)}{{ }_{(N)} h_{l+n-1}} \frac{A_{l, N-l+1}^{(n)}}{B_{l, N-l+1}} .
$$

Passing to the limit $N \rightarrow \infty$ we get the decoupled recursion (21) provided that the limits $\lim _{N \rightarrow \infty} A_{l, N-l+1}^{(i)} / B_{l, N-l+1}$ do exist for $i=0,1, \cdots, n$.

To prove our assertion we utilize that the invariant measure $\left(x_{k}\right)_{k \geq 0}$ of the irreducible and recurrent Markov chain (1) is strictly positive and satisfies the recurrence relation (4) which can be rewritten as

$$
\begin{aligned}
& \frac{P_{k, k+n} \cdot x_{k}}{P_{k+n+1, k+n} \cdot x_{k+n+1}}+\frac{P_{k+1, k+n} \cdot x_{k+1}}{P_{k+n+1, k+n} \cdot x_{k+n+1}}+\cdots+\frac{P_{k+n-1, k+n} \cdot x_{k+n-1}}{P_{k+n+1, k+n} \cdot x_{k+n+1}}+1 \\
& =\frac{\left(1-P_{k+n, k+n}\right) x_{k+n}}{P_{k+n+1, k+n} \cdot x_{k+n+1}} \quad(k=0,1,2, \cdots) .
\end{aligned}
$$

Define $\rho_{k}=\frac{x_{k+n}}{x_{k+n+1}}$ for $k=0,1,2, \cdots$ Then (30) becomes equivalent to

$$
\begin{aligned}
& \left|a_{k}^{(1)} \rho_{k} \rho_{k-1} \cdots \rho_{k-n}\right|+\left|a_{k}^{(2)} \rho_{k} \rho_{k-1} \cdots \rho_{k-n+1}\right| \\
& +\cdots+\left|a_{k}^{(n)} \rho_{k} \rho_{k-1}\right|+1=\left|b_{k} \rho_{k}\right| \quad(k=0,1,2, \cdots)
\end{aligned}
$$

implying that the GCFs

$$
\left[\begin{array}{ccc}
a_{l}^{(1)} \rho_{l} \rho_{l-1} \cdots \rho_{l-n} & a_{l+1}^{(1)} \rho_{l+1} \rho_{l} \cdots \rho_{l-n+1} & \cdots \\
a_{l}^{(2)} \rho_{l} \rho_{l-1} \cdots \rho_{l-n+1} & a_{l+1}^{(2)} \rho_{l+1} \rho_{l} \cdots \rho_{l-n+2} & \cdots \\
\vdots & \vdots & \\
a_{l}^{(n)} \rho_{l} \rho_{l-1} & a_{l+1}^{(n)} \rho_{l+1} \rho_{l} & \cdots \\
b_{l} \rho_{l} & b_{l+1} \rho_{l} & \cdots
\end{array}\right]
$$

converge for all $l \geq 0$ by means of Theorem 2. From Theorem 3, we then conclude that the same is true for the GCFs (8).

Theorem 2 says that the numerical calculation of the invariant measures of $X$ 
can be reduced to Van der Cruyssen's algorithm with (23) as initial conditions.

Remark. It is important that the statement of Theorem 4 consists of two parts:

- The invariant measure is dominated by other solutions of the difference Equation (4).

- By means of GCFs, we are able to construct a decoupled recursion which is not affected to numerical instability.

Our main goal was the derivation of the first statement. Although the existence of dominating solutions has been pointed out in specific examples (e.g. ([8], p. 167) where the exact solutions of the difference equations are known), to our knowledge, no statement in this generality has been published. Since the desired solution being dominated directly implies numerical instability, the system $x=x P$ should not be solved by forward computation. Note, that up to some slight modifications concerning the boundary conditions, the truncated system (22) yields the same difference Equation (4) for $k=0,1, \cdots, N-n-1$. Thus, forward computation could be applied to (22), too, but at least for large $N$, the result of numerical instability and hence, the recommendation not to use forward computation, holds for (22) as well.

The observation of inherent numerical instability of (4) is essential since the transition structure under consideration arises in many practical applications, e.g. state-dependent queueing systems with bulk arrivals, and it is also valid in a more general context, e.g. as an approximation to state-dependent variants of the traditional $G / M / 1$ queueing model.

Basically, we have exploited that the solutions of the truncated system (22) converge to invariant measures as $N \rightarrow \infty$. For Markov chains with a general transition structure, there is no way of solving $x=x P$ directly (in particular, forward computation cannot be applied if $P_{k l}>0$ for some $\left.k<l-1\right)$. In this situation, Seneta [20] [21] [22] as well as Golub and Seneta [23] already recommended to use the convergence of the solutions of the truncated system (22) to the invariant measure for numerical issues. Furthermore, Seneta [22] discussed numerical aspects (in terms of the condition number) of the finite system (22) and stated numerical stability of Gaussian elimination or LU decomposition. The above comment concerning forward computation as a solution method for (22) emphasizes on the fact that it is important to solve this finite system in an appropriate way. The GCF-based algorithm can be interpreted as a combination of building up the truncated system (22), and then applying a modification of Gaussian elimination procedure. Therefore, it follows both steps recommended in the literature cited above and represents the complete procedure in a combined mathematical form, which is interesting from a structural point of view.

\section{Continuous-Time Markov Chains}

The results of the preceding chapter can easily be extended to continuous-time Markov chains $Y=\left(Y_{t}\right)_{t \geq 0}$ generated by a conservative, irreducible and regular 
Q-matrix of the form

$$
Q=\left[\begin{array}{ccccccccc}
q_{00} & q_{01} & q_{02} & \cdots & q_{0 n} & O & O & \cdots & \cdots \\
q_{10} & q_{11} & q_{12} & \cdots & q_{1 n} & q_{1, n+1} & O & \cdots & \cdots \\
O & q_{21} & q_{22} & \cdots & q_{2 n} & q_{2, n+1} & q_{2, n+2} & O & \cdots \\
O & O & q_{32} & \cdots & q_{3 n} & q_{3, n+1} & q_{3, n+2} & q_{3, n+3} & \\
\ddots & \ddots & \ddots & & \ddots & \ddots & \ddots & \ddots & \ddots
\end{array}\right]
$$

where $n \geq 1$ and $q_{k, k+n} \neq 0$ for at least one $k \in \mathbb{N}_{0}$. Notice that the irreducibility of $Y$ implies

$$
q_{k, k-1} \neq 0 \text { for } k=1,2, \cdots
$$

If $Y$ is positive recurrent, the limits

$$
\pi_{k}=\lim _{t \rightarrow \infty} P\left(Y_{t}=k \mid Y_{0}=i\right) \quad(k=0,1,2, \cdots)
$$

exist independently of the initial state and build up a probability distribution which is strictly positive. For the construction of a continuous-time Markov process from its infinitesimal properties the reader is referred to Reuter [24] and Kendall and Reuter [25]. Now let ${ }_{(N)} Q$ be the $(N+1) \times(N+1)$ northwest corner truncation of $Q$. If $Y$ is regular and positive recurrent, then the finite system

$$
{ }_{(N)} Z_{(N)} Q={ }_{(N)} e_{0}
$$

has a unique solution ${ }_{(N)} Z=\left({ }_{(N)} Z_{0},{ }_{(N)} Z_{1}, \cdots,{ }_{(N)} Z_{N}\right)$ satisfying

$$
\lim _{N \rightarrow \infty} \frac{(N) Z_{k}}{{ }_{(N)} Z_{0}}=\frac{\pi_{k}}{\pi_{0}} \quad(k=0,1,2, \cdots),
$$

see Tweedie [26]. Now let

$$
\begin{gathered}
c_{k}^{(i)}=-\frac{q_{k+i-1, k+n}}{q_{k+n+1, k+n}}(i=1, \cdots, n, \quad k=0,1,2, \cdots), \\
d_{k}=-\frac{q_{k+n, k+n}}{q_{k+n+1, k+n}} \quad(k=0,1,2, \cdots) .
\end{gathered}
$$

By substituting (31) into (32) it is seen that ${ }_{(N)} Z$ satisfies the $(n+1)$ th order homogeneous linear difference equation

$$
\pi_{k+n+1}=d_{k} \pi_{k+n}+c_{k}^{(n)} \pi_{k+n-1}+\cdots+c_{k}^{(1)} \pi_{k} \quad(k=0,1,2, \cdots)
$$

for $k=0,1, \cdots, N-n$ and the boundary conditions

$$
\left.\begin{array}{c}
q_{00(N)} z_{0}+q_{10(N)} z_{1}=1 \\
q_{01(N)} z_{0}+q_{11(N)} z_{1}+q_{21(N)} z_{2}=0 \\
\vdots \\
q_{0, n-1(N)} z_{0}+q_{1, n-1(N)} z_{1}+\cdots+q_{n, n-1(N)} z_{n}=0
\end{array}\right\}
$$

By replacing (33) with (18) and (34) with (19), it is readily seen that the scheme (35)-(37) formally coincides with that of the discrete time case. Hence (26)-(29) also hold for ${ }_{(N)} Z_{l}$. To prove convergence of the associated GCFs 


$$
\left[\begin{array}{c}
\Lambda_{l}^{(1)} \\
\vdots \\
\Lambda_{l}^{(n)}
\end{array}\right]=\left[\begin{array}{cccc}
c_{l}^{(1)} & c_{l+1}^{(1)} & c_{l+2}^{(1)} & \cdots \\
\vdots & \vdots & \vdots & \\
c_{l}^{(n)} & c_{l+1}^{(n)} & c_{l+2}^{(n)} & \cdots \\
d_{l} & d_{l+1} & d_{l+2} & \cdots
\end{array}\right] \quad(l=0,1,2, \cdots)
$$

we use the same arguments as in the proof of Theorem 4. Notice that $\left(\pi_{k}\right)_{k=0}^{\infty}$ satisfies (35). Rearranging yields

$$
-c_{k}^{(1)} \frac{\pi_{k}}{\pi_{k+n+1}}-\cdots-c_{k}^{(n)} \frac{\pi_{k+n-1}}{\pi_{k+n+1}}+1=d_{k} \frac{\pi_{k+n}}{\pi_{k+n+1}} \quad(k=0,1,2, \cdots) .
$$

Define $\gamma_{k}=\frac{\pi_{k+n}}{\pi_{k+n+1}}$ for $k=0,1,2, \cdots$. Since $-c_{k}^{(i)}>0$ for $i=1, \cdots, n, k \geq 0$ and $d_{k}>0$ for $k \geq 0$, (39) is equivalent to

$$
\begin{aligned}
& \left|c_{k}^{(i)} \gamma_{k} \gamma_{k-1} \cdots \gamma_{k-n}\right|+\left|c_{k}^{(2)} \gamma_{k} \gamma_{k-1} \cdots \gamma_{k-n+1}\right|+\cdots+\left|c_{k}^{(n)} \gamma_{k} \gamma_{k-1}\right|+1 \\
& =\left|d_{k} \gamma_{k}\right| \quad(k=0,1,2, \cdots) .
\end{aligned}
$$

By Theorem 3, the GCF

$$
\left[\begin{array}{ccc}
c_{l}^{(1)} \gamma_{l} \gamma_{l-1} \cdots \gamma_{l-n} & c_{l+1}^{(1)} \gamma_{l+1} \gamma_{l} \cdots \gamma_{l-n+1} & \cdots \\
c_{l}^{(2)} \gamma_{l} \gamma_{l-1} \cdots \gamma_{l-n+1} & c_{l+1}^{(2)} \gamma_{l+1} \gamma_{l} \cdots \gamma_{l-n+2} & \cdots \\
\vdots & \vdots & \\
c_{l}^{(n)} \gamma_{l} \gamma_{l-1} & c_{l+1}^{(n)} \gamma_{l+1} \gamma_{l} & \cdots \\
d_{l} \gamma_{l} & d_{l+1} \gamma_{l} & \cdots
\end{array}\right]
$$

then converges for all $l \geq 0$. Hence the same is true for the GCFs (38). Summarizing we arrive at the following theorem.

Theorem 5. Let $c_{k}^{(i)}, d_{k}$ be defined as in (33) and (34), respectively. The limits (38) exist for all $l \geq 0$ if the corresponding Markov process is irreducible, regular and positive recurrent. In case of convergence the limiting distribution $\left(\pi_{k}\right)_{k=0}^{\infty}$ is a dominated solution of (35) and satisfies the decoupled recursion.

$$
\pi_{l+n}=-\Lambda_{l}^{(n)} \pi_{l+n-1}-\cdots-\Lambda_{l}^{(1)} \pi_{l} \quad(l=0,1,2, \cdots) .
$$

For executing (40), we make use of Van der Cruyssen's algorithm with (36) as initial conditions. The resulting sequence $\left({ }_{(N)} Z_{k}\right)_{k=0}^{N}$ can be normalized such that $\sum_{k=0}^{N}{ }_{(N)} Z_{k}=1$.

Remark. As for discrete-time Markov chains, the key statement of Theorem 5 is that the invariant measure is a dominated solution of the difference equation (35). Again, the GCFs additionally combine the two-step method consisting of considering the truncated system (32) and applying Gaussian elimination or LU decomposition.

\section{Numerical Examples}

As an example we consider the continuous-time Markov chain $Y=\left(Y_{t}\right)_{t \geq 0}$ generated by the Q-matrix $Q=\left(q_{i j}\right)_{0 \leq i, k<\infty}$, where 


$$
q_{i k}= \begin{cases}\frac{a_{0} a_{1}}{\gamma^{i}} & k=i-1, i \geq 1 \\ \frac{a_{1}-a_{0}-1}{\gamma^{i}}, & k=i+1 \\ \gamma^{i}, & k=i+2 \\ -\frac{a_{1}-a_{0}}{\gamma^{i}}, & k=i=0 \\ -\frac{a_{0} a_{1}+a_{1}-a_{0}}{\gamma^{i}}, & k=i \geq 1 \\ 0, & \text { elsewhere }\end{cases}
$$

with parameters $a_{0}>\gamma>1$ and $a_{1} \geq a_{0}+1$. Such a Markov chain may be interpreted as a model for a single-server queueing system with state-dependent arrival and service rates, and with customers arriving in groups of size 1 or 2 .

It is easy to check that $Q$ is irreducible. To prove that $Y$ is regular and positive recurrent we make use of Tweedie's [27] criterion. The condition is to find some $\varepsilon>0$ and a non-negative sequence $z=\left(z_{0}, z_{1}, \cdots\right)$ satisfying $z_{i} \rightarrow \infty$ as $i \rightarrow \infty$ and

$$
\sum_{k=0}^{\infty} q_{i k} z_{k} \leq-\varepsilon
$$

for almost all $i$. By substituting (41) into (42) we get

$$
\frac{1}{\gamma^{i}} z_{i+2}+\frac{a_{1}-a_{0}-1}{\gamma^{i}} z_{i+1}-\frac{a_{1}-a_{0}}{\gamma^{i}} z_{i}+\frac{a_{0} a_{1}}{\gamma^{i}} z_{i-1} \leq-\varepsilon
$$

for almost all $i$. The substitution $z_{i}=\gamma^{i}$ for $i=0,1,2, \cdots$ implies the condition

$$
\frac{(\gamma-1)\left(\gamma-a_{0}\right)\left(\gamma+a_{1}\right)}{\gamma} \leq-\varepsilon
$$

for almost all $i$ which is true because of $a_{0}>\gamma>1$.

Under these conditions there exists a unique invariant measure of $Y$ (up to multiplication by a constant), which satisfies the following set of equations:

$$
\begin{gathered}
\frac{a_{0} a_{1}}{\gamma} \pi_{1}-\left(a_{1}-a_{0}\right) \pi_{0}=0, \\
\frac{a_{0} a_{1}}{\gamma^{2}} \pi_{2}-\frac{a_{0} a_{1}+a_{1}-a_{0}}{\gamma} \pi_{1}+\left(a_{1}-a_{0}-1\right) \pi_{0}=0
\end{gathered}
$$

and

$$
\frac{a_{0} a_{1}}{\gamma^{k+3}} \pi_{k+3}-\frac{a_{0} a_{1}+a_{1}-a_{0}}{\gamma^{k+2}} \pi_{k+2}+\frac{a_{1}-a_{0}-1}{\gamma^{k+1}} \pi_{k+1}+\frac{1}{\gamma^{k}} \pi_{k}=0
$$

or, equivalently,

$$
a_{0} a_{1} \pi_{k+3}-\left(a_{0} a_{1}+a_{1}-a_{0}\right) \gamma \pi_{k+2}+\left(a_{1}-a_{0}-1\right) \gamma^{2} \pi_{k+1}+\gamma^{3} \pi_{k}=0
$$

for $k=0,1,2, \cdots$. (45) leads to the recursion 


$$
\pi_{k+3}=\frac{\gamma\left(a_{0} a_{1}+a_{1}-a_{0}\right) \pi_{k+2}-\gamma^{2}\left(a_{1}-a_{0}-1\right) \pi_{k+1}-\gamma^{3} \pi_{k}}{a_{0} a_{1}}
$$

which is used for forward computation.

Since (45) is a homogenous linear difference equation with constant coefficients a fundamental set of solutions can easily be derived from the roots of its characteristic equation. A simple manipulation yields the following set of linearly independent solutions:

$$
\left(\left(\frac{\gamma}{a_{0}}\right)^{k}\right)_{k=0}^{\infty},\left(\left(-\frac{\gamma}{a_{1}}\right)^{k}\right)_{k=0}^{\infty},\left(\gamma^{k}\right)_{k=0}^{\infty} .
$$

The desired invariant measure $\left(\pi_{k}\right)_{k=0}^{\infty}$ of $Y$ with initial value $\pi_{0}=1$ is a linear combination of the first two solutions, that is:

$$
\pi_{k}=\frac{a_{1}}{a_{0}+a_{1}}\left(\frac{\gamma}{a_{0}}\right)^{k}+\frac{a_{0}}{a_{0}+a_{1}}\left(-\frac{\gamma}{a_{1}}\right)^{k} \quad(k=0,1,2, \cdots) .
$$

Therefore, choosing $\gamma>1$ yields that there is a geometrically increasing solution of (46), which explains why forward computation cannot cope with this problem, whereas the GCF-based algorithm becomes a stable procedure. This

\begin{tabular}{|c|c|c|c|}
\hline$k$ & Forward computation & GCF based solution & Exact solution \\
\hline 0 & 1 & 1 & 1 \\
\hline 1 & 0.171429 & 0.171429 & 0.171429 \\
\hline 2 & 0.286531 & 0.286531 & 0.286531 \\
\hline 3 & 0.0932012 & 0.0932012 & 0.0932012 \\
\hline 4 & 0.0896566 & 0.0896566 & 0.0896566 \\
\hline 5 & 0.0393357 & 0.0393357 & 0.0393357 \\
\hline 17 & $9.85074 \times 10^{-5}$ & $9.85075 \times 10^{-5}$ & $9.85075 \times 10^{-5}$ \\
\hline 18 & $5.93420 \times 10^{-5}$ & $5.93424 \times 10^{-5}$ & $5.93424 \times 10^{-5}$ \\
\hline 19 & $3.55023 \times 10^{-5}$ & $3.55035 \times 10^{-5}$ & $3.55035 \times 10^{-5}$ \\
\hline 20 & $2.13423 \times 10^{-5}$ & $2.13458 \times 10^{-5}$ & $2.13458 \times 10^{-5}$ \\
\hline 21 & $1.27782 \times 10^{-5}$ & $1.27888 \times 10^{-5}$ & $1.27888 \times 10^{-5}$ \\
\hline 22 & $7.64963 \times 10^{-6}$ & $7.68128 \times 10^{-6}$ & $7.68128 \times 10^{-6}$ \\
\hline 23 & $4.51039 \times 10^{-6}$ & $4.60533 \times 10^{-6}$ & $4.60533 \times 10^{-6}$ \\
\hline 24 & $2.47987 \times 10^{-6}$ & $2.76467 \times 10^{-6}$ & $2.76467 \times 10^{-6}$ \\
\hline 25 & $8.03766 \times 10^{-7}$ & $1.65817 \times 10^{-6}$ & $1.65817 \times 10^{-6}$ \\
\hline 26 & $-1.56804 \times 10^{-6}$ & $9.95173 \times 10^{-7}$ & $9.95173 \times 10^{-7}$ \\
\hline 27 & $-7.09265 \times 10^{-6}$ & $5.96988 \times 10^{-7}$ & $5.96988 \times 10^{-7}$ \\
\hline 28 & $-2.27107 \times 10^{-5}$ & $3.58242 \times 10^{-7}$ & $3.58242 \times 10^{-7}$ \\
\hline 29 & $-6.89918 \times 10^{-5}$ & $2.14924 \times 10^{-7}$ & $2.14924 \times 10^{-7}$ \\
\hline 30 & -0.000207491 & $1.28964 \times 10^{-7}$ & $1.28964 \times 10^{-7}$ \\
\hline 40 & -12.2598 & $7.79771 \times 10^{-10}$ & $7.79771 \times 10^{-10}$ \\
\hline 50 & $-723,927$ & $4.71497 \times 10^{-12}$ & $4.71497 \times 10^{-12}$ \\
\hline
\end{tabular}
effect is reflected in Table 1 and Table 2, where data type double in $\mathrm{C}++$ was used.

Table 1. Numerical values for $\gamma=3, a_{0}=5, a_{1}=7$. 
Table 2. Numerical values for $\gamma=9, a_{0}=10, a_{1}=12$.

\begin{tabular}{|c|c|c|c|}
\hline$k$ & Forward computation & GCF based solution & Exact solution \\
\hline 0 & 1 & 1 & 1 \\
\hline 1 & 0.15 & 0.15 & 0.15 \\
\hline 2 & 0.6975 & 0.6975 & 0.6975 \\
\hline 3 & 0.205875 & 0.205875 & 0.205875 \\
\hline 4 & 0.501694 & 0.501694 & 0.501694 \\
\hline 5 & 0.21422 & 0.21422 & 0.21422 \\
\hline 13 & 0.127849 & 0.127848 & 0.127848 \\
\hline 14 & 0.132884 & 0.132882 & 0.132882 \\
\hline 15 & 0.106256 & 0.10623 & 0.10623 \\
\hline 16 & 0.105863 & 0.10563 & 0.10563 \\
\hline 17 & 0.0896527 & 0.0875497 & 0.0875497 \\
\hline 18 & 0.10336 & 0.0844324 & 0.0844324 \\
\hline 19 & 0.242111 & 0.0717609 & 0.0717609 \\
\hline 20 & 1.60091 & 0.067756 & 0.067756 \\
\hline 21 & 13.857 & 0.058602 & 0.058602 \\
\hline 22 & 124.24 & 0.0545256 & 0.0545256 \\
\hline 23 & 1117.72 & 0.0477352 & 0.0477352 \\
\hline 24 & $10,059.1$ & 0.0439651 & 0.0439651 \\
\hline 25 & $90,531.2$ & 0.038816 & 0.038816 \\
\hline
\end{tabular}

Consider next the $M / M / 1$ queueing system, in which the customers arrive according to a Poisson stream with rate $\lambda>0$ and in which the successive service times are stochastically independent and exponentially distributed with parameter $\mu>0$. For this system it is known that the queueing process $L=(L(t))_{t \geq 0}$ which counts the number of customers in line, forms a homogeneous continuous time Markov chain with state space $\mathbb{N}_{0}=\{0,1,2, \cdots\}$ being regular and positive recurrent for $\rho=\frac{\lambda}{\mu}<1$, see [28]. The model is convenient to get clear about the mechanism of our approach.

The invariant measure $\left(\pi_{k}\right)_{k=0}^{\infty}$ of $L$ meets the second order linear difference equation

$$
\lambda \pi_{k-1}-(\lambda+\mu) \pi_{k}+\mu \pi_{k+1}=0 \quad(k=1,2, \cdots)
$$

subject to the side conditions

$$
\pi_{0}=1 \text { and }-\lambda \pi_{0}+\mu \pi_{1}=0 .
$$

In principle, $\left(\pi_{k}\right)_{k=0}^{\infty}$ could be computed by forward computation, that is

$$
\begin{gathered}
\pi_{0}=1, \\
\pi_{1}=\rho \pi_{0},
\end{gathered}
$$




$$
\pi_{k+1}=(\rho+1) \pi_{k}-\rho \pi_{k-1} \quad(k=1,2, \cdots)
$$

where $\rho=\frac{\lambda}{\mu}<1$.

A simple induction argument shows that $\pi_{k}=\rho^{k}$ for $k=0,1,2, \cdots$. Another solution of (47) is given by $\tilde{\pi}_{k}=1$ for $k=0,1,2, \cdots$, implying that $\left(\pi_{k}\right)_{k=0}^{\infty}$ becomes a dominated solution of (47), as claimed. Therefore $\left(\pi_{k}\right)_{k=0}^{\infty}$ can also be characterized by the decoupled recursion (40), i.e.

$$
\pi_{k+1}=-\Lambda_{k}^{(1)} \pi_{k} \quad(k=0,1,2, \cdots) .
$$

Substituting of (48) into (47) leads to

$$
\Lambda_{k-1}^{(1)}=-\frac{1}{\left(1+\rho^{-1}\right)+\rho^{-1} \Lambda_{k}^{(1)}} \quad(k=1,2, \cdots) .
$$

By truncating this infinite continued fraction scheme at a certain level $k=N$, we get approximants for the desired coefficients $\Lambda_{k}^{(1)}$. A numerical example is given in Table 3 . In this example the effect of numerical instability is comparatively small.

\section{Conclusion and Further Research}

For Markov chains with a specific transition structure, we have proven that the

Table 3. Numerical values for the $\mathrm{M} / \mathrm{M} / 1$ queueing system for $\rho=\frac{1}{3}$.

\begin{tabular}{cccc}
\hline$k$ & Forward computation & GCF based solution & Exact solution \\
\hline 0 & 1 & 1 & 1 \\
1 & 0.333333 & 0.3333 & 0.3333 \\
2 & 0.111111 & 0.1111 & 0.1111 \\
3 & 0.03704 & 0.03704 & 0.03704 \\
4 & 0.01235 & 0.01235 & 0.01235 \\
5 & 0.004115 & 0.004115 & 0.004115 \\
6 & 0.001372 & 0.001372 & 0.001372 \\
7 & $4.572 \times 10^{-4}$ & $4.572 \times 10^{-4}$ & $4.572 \times 10^{-4}$ \\
8 & $1.524 \times 10^{-4}$ & $1.524 \times 10^{-4}$ & $1.524 \times 10^{-4}$ \\
10 & $1.694 \times 10^{-5}$ & $1.694 \times 10^{-5}$ & $1.694 \times 10^{-5}$ \\
12 & $1.882 \times 10^{-6}$ & $1.882 \times 10^{-6}$ & $1.882 \times 10^{-6}$ \\
15 & $6.969 \times 10^{-8}$ & $6.969 \times 10^{-8}$ & $6.969 \times 10^{-8}$ \\
20 & $2.868 \times 10^{-10}$ & $2.868 \times 10^{-10}$ & $2.868 \times 10^{-10}$ \\
25 & $1.180 \times 10^{-12}$ & $1.180 \times 10^{-12}$ & $1.180 \times 10^{-12}$ \\
30 & $4.870 \times 10^{-15}$ & $4.857 \times 10^{-15}$ & $4.857 \times 10^{-15}$ \\
40 & $1.312 \times 10^{-17}$ & $8.225 \times 10^{-20}$ & $8.225 \times 10^{-20}$ \\
50 & $1.304 \times 10^{-17}$ & $1.393 \times 10^{-24}$ & $1.393 \times 10^{-24}$ \\
90 & $1.304 \times 10^{-17}$ & $1.146 \times 10^{-43}$ & $1.146 \times 10^{-43}$ \\
\hline & & & \\
\hline
\end{tabular}


invariant measure is a dominated solution of the corresponding steady-state recurrence relations, and therefore, it cannot be calculated by forward computation. As a by-product of our considerations, a GCF-based method for computing the invariant measure in a numerically stable way has been established. The effects of numerical instability of the original recurrence relations as well as the stability of the decoupled version have been illustrated by numerical examples. In some way, the GCF-based approach is similar to previously recommended methods (truncate the infinite system and solve the truncated one by Gaussian elimination or LU decomposition), but represents the steps in a combined mathematical form. Note that in previous literature, the generality of the transition structure did not allow forward computation, and hence, the question of instability of this method did not arise.

At no point of our consideration, we used stochasticity of $P$ (or the corresponding property of conservativity of $Q$ ) explicitly. Therefore, it seems reasonable to extend our results to more general infinite systems $S x=0$ of linear equations, where

$$
S=\left(\begin{array}{cccccccc}
S_{00} & S_{01} & & & & & & \\
S_{10} & S_{11} & S_{12} & & & & & \\
S_{20} & S_{21} & S_{22} & S_{23} & & & & \\
& \cdots & \cdots & \cdots & \ddots & \ddots & & \\
S_{n 0} & S_{n 1} & \cdots & \cdots & S_{n n} & S_{n, n+1} & & \\
& S_{n+1,0} & S_{n+1,1} & \cdots & \cdots & S_{n+1, n+1} & S_{n+1, n+2} & \\
& & \ddots & \ddots & \cdots & \cdots & \ddots & \ddots
\end{array}\right) .
$$

In the context of computing invariant measures of Markov chains, we have $S=I-P^{\mathrm{T}}$ or $S=Q^{\mathrm{T}}$, respectively. In the general case, the main steps of the proofs of Theorem 4 or Theorem 5 can be restated in an abstract context as follows: If $s_{k k}>0$ and $s_{k, k+1}<0$ for $k=0,1,2, \cdots$, and $s_{k l} \leq 0$ for $k=1,2,3, \cdots$ and $l<k$, and if there is a strictly positive solution $x$ of $S x=0$, all GCFs involved in the algorithmic procedure converge. If additionally, we have $\lim _{N \rightarrow \infty} \frac{(N) X_{n}}{{ }_{(N)} X_{0}}=\frac{X_{n}}{X_{0}}$ for all $n=0,1,2, \cdots$ for the solutions ${ }_{(N)} X$ of ${ }_{(N)} X \cdot{ }_{(N)} S={ }_{(N)} e_{0}$, then the GCF-based algorithm converges to $X$ as $N$ tends to infinity, see [29]. Usually, these assumptions are met in the context of computing Perron-Frobenius eigenvectors for infinite non-negative matrices with some communication structure (e.g. irreducibility, see [30] for more details). In the context of discrete-time Markov chains, invariant measures are right eigenvectors for the Perron-Frobenius eigenvalue 1, whereas left eigenvectors are related to hitting probabilities (or absorption probabilities). Therefore, an extension of our considerations could refer to the task of computing these probabilities under appropriate assumptions on the transition structure. Further generalizations could deal with the following generalizations:

- In the tridiagonal case (that is, $n=1$ ), a generalization to block-matrices 
(that is, the corresponding Markov chain is a quasi-birth-death process) by means of matrix-valued continued fractions has been studied in [31]. The resulting algorithm is similar to the matrix-analytic methods studied in [32] [33]. An intuitive combination is the study of transition probability matrices of the form (1) where all $P_{k l}$ are matrices. For practical issues, it would be desirable to find a memory-efficient algorithm for computing stationary expectations. For quasi-birth-death processes (that is, block-tridiagonal transition matrices), such a method has been suggested in [34].

- Instead of banded matrices, we could consider upper Hessenberg matrices $P$ (or lower Hessenberg matrices $S$ ). Then the difference equation (4) of order $n$ is replaced by a difference equation of infinite order (sometimes referred to as sum equation). Furthermore, arbitrary lower bandwidthes for $P$ could be considered (as in [9], where the upper bandwidth was restricted to 1).

\section{Acknowledgements}

We acknowledge support by Open Access Publishing Fund of Clausthal University of Technology.

\section{References}

[1] Karlin, S. and Taylor, H.M. (1981) A Second Course in Stochastic Processes. Academic Press, New York.

[2] Miller, K.S. (1968) Linear Difference Equations. Benjamin, New York.

[3] Cash, J.R. (1980) A Note on the Numerical Solution of Linear Recurrence Relations. Numerische Mathematik, 34, 371-386. https://doi.org/10.1007/BF01403675

[4] Gautschi, W. (1967) Computational Aspects of Three-Term Recurrence Relations. SIAM Review, 9, 24-82. https://doi.org/10.1137/1009002

[5] Gautschi, W. (1972) Zur Numerik rekurrenter Relationen. Computing, 9, 107-126. https://doi.org/10.1007/BF02236961

[6] Lozier, S.W. (1980) Numerical Solution of Linear Difference Equations. Report NBSIR 80-1976, National Bureau of Standards, US Dept. of Commerce, Washington DC. https://doi.org/10.6028/NBS.IR.80-1976

[7] Mattheij, R.M.M. (1980) Characterization of Dominant and Dominated Solutions of Linear Recursions. Numerische Mathematik, 35, 421-442. https://doi.org/10.1007/BF01399009

[8] Wimp, J. (1984) Computation with Recurrence Relations. Pitman, Boston.

[9] Hanschke, T. (1992) Markov Chains and Generalized Continued Fractions. Journal of Applied Probability, 29, 838-849. https://doi.org/10.1017/S0021900200043710

[10] Jacobi, C.G.J. (1868) Allgemeine Theorie der kettenbruchähnlichen Algorithmen, in welchen jede Zahl aus drei vorhergehenden gebildet wird. [General Theory of Continued-Fraction-Type Algorithms in Which Every Number Is Generated by the Three Preceding Ones.] Journal für die reine und angewandte Mathematik, 69, 29-64. https://doi.org/10.1515/crll.1868.69.29

[11] Perron, O. (1907) Grundlagen für eine Theorie des Jacobischen Kettenbruchalgorithmus. [Fundamentals for a Theory of Jacobi's Continued-Fraction Algorithm.] Mathematische Annalen, 64, 1-76. https://doi.org/10.1007/BF01449880 
[12] Perron, O. (1907) Über die Konvergenz der Jacobi-Kettenalgorithmen mit komplexen Elementen. [On the Convergence of Jacobi's Continued-Fraction Algorithms with Complex Elements.] Sitzungsber. Akad. München, 37, 401-482.

[13] Van der Cruyssen, P. (1979) Linear Difference Equations and Generalized Continued Fractions. Computing, 22, 269-278. https://doi.org/10.1007/BF02243567

[14] Perron, O. (1909) Über lineare Differenzen-und Differentialgleichungen. [On Linear Difference and Differential Equations.] Mathematische Annalen, 66, 446-487. https://doi.org/10.1007/BF01450044

[15] Hanschke, T. (1991) Über die Minimallösung der Poincare-Perronschen Differenzengleichung. [On the Minimal Solution of the Poincaré-Perron Difference Equation.] Monatshefte für Mathematik, 112, 281-295. https://doi.org/10.1007/BF01351769

[16] Hanschke, T. (1998) Ein verallgemeinerter Jacobi-Perron-Algorithmus zur Reduktion linearer Differenzengleichungssysteme. [A Generalized Jacobi-Perron Algorithm for the Reduction of Systems of Linear Difference Equations.] Monatshefte für Mathematik, 126, 287-311. https://doi.org/10.1007/BF01299054

[17] Levrie, P. and Bultheel, A. (1996) Matrix Continued Fractions Related to First-Order Linear Recurrence Systems. Electronic Transactions on Numerical Analysis, 4, 46-63.

[18] Levrie, P. (1986) Pringsheim's Theorem for Generalized Continued Fractions. Journal of Computational and Applied Mathematics, 14, 439-445.

[19] de Bruin, M.G. (1978) Convergence of Generalized C-Fractions. Journal of Approximation Theory, 24, 177-207. https://doi.org/10.1016/0021-9045(78)90023-0

[20] Seneta, E. (1967) Finite Approximations to Infinite Non-Negative Matrices. Proceedings of the Cambridge Philosophical Society, 63, 983-992.

https://doi.org/10.1017/S0305004100042006

[21] Seneta, E. (1968) Finite Approximations to Infinite Non-Negative Matrices II. Proceedings of the Cambridge Philosophical Society, 64, 465-470. https://doi.org/10.1017/S0305004100043061

[22] Seneta, E. (1980) Computing the Stationary Distribution for Infinite Markov Chains. Linear Algebra and Its Applications, 34, 259-267.

[23] Golub, G. and Seneta, E. (1974) Computation of the Stationary Distribution of an Infinite Stochastic Matrix of Special Form. Bulletin of the Australian Mathematical Society, 10, 255-261. https://doi.org/10.1017/S0004972700040867

[24] Reuter, G.E.H. (1957) Denumerable Markov Processes and the Associated Contraction Semigroup on L. Acta Mathematica, 97, 1-46. https://doi.org/10.1007/BF02392391

[25] Kendall, D.G. and Reuter, G.E.H. (1957) The Calculation of the Ergodic Projection for Markov Chains and Processes with a Countable Infinity of States. Acta Mathematica, 97, 103-144. https://doi.org/10.1007/BF02392395

[26] Tweedie, R.L. (1973) The Calculation of Limit Probabilities for Denumerable Markov Processes from Infinitesimal Properties. Journal of Applied Probability, 10, 84-99. https://doi.org/10.1017/S0021900200042108

[27] Tweedie, R.L. (1975) Sufficient Conditions for Regularity, Recurrence and Ergodicity of Markov Processes. Mathematical Proceedings of the Cambridge Philosophical Society, 78, 125-136. https://doi.org/10.1017/S0305004100051562

[28] Gross, D., Shortle, J.F., Thompson, J.M. and Harris, C.M. (2008) Fundamentals of Queueing Theory. 4th Edition, Wiley, New Jersey.

https://doi.org/10.1002/9781118625651 
[29] Baumann, H. (2017) Generalized Continued Fractions: Definitions, Convergence and Applications to Markov Chains. Habilitationsschrift. Clausthal University of Technology, Clausthal-Zellerfeld.

[30] Seneta, E. (1981) Non-Negative Matrices and Markov Chains. Springer, New York. https://doi.org/10.1007/0-387-32792-4

[31] Hanschke, T. (1999) A Matrix Continued Fraction Algorithm for the Multiserver Repeated Order Queue. Mathematical and Computer Modelling, 30, 159-170.

[32] Baumann, H. and Sandmann, W. (2010) Numerical Solution of Level Dependent Quasi-Birth-and-Death Processes. Procedia Computer Science, 1, 1561-1569. https://doi.org/10.1016/j.procs.2010.04.175

[33] Bright, L. and Taylor, P.G. (1995) Calculating the Equilibrium Distribution in Level Dependent Quasi-Birth-and-Death Processes. Communication in Statistics. Stochastic Models, 11, 497-525. https://doi.org/10.1080/15326349508807357

[34] Baumann, H. and Sandmann, W. (2013) Computing Stationary Expectations in Level-Dependent QBD Processes. Journal of Applied Probability, 50, 151-165. https://doi.org/10.1017/S0021900200013176

\section{Scientific Research Publishing}

Submit or recommend next manuscript to SCIRP and we will provide best service for you:

Accepting pre-submission inquiries through Email, Facebook, LinkedIn, Twitter, etc. A wide selection of journals (inclusive of 9 subjects, more than 200 journals) Providing 24-hour high-quality service User-friendly online submission system Fair and swift peer-review system Efficient typesetting and proofreading procedure Display of the result of downloads and visits, as well as the number of cited articles Maximum dissemination of your research work

Submit your manuscript at: http://papersubmission.scirp.org/

Or contact am@scirp.org 